Document Number: SET 2014-0037

412TW-PA-14270
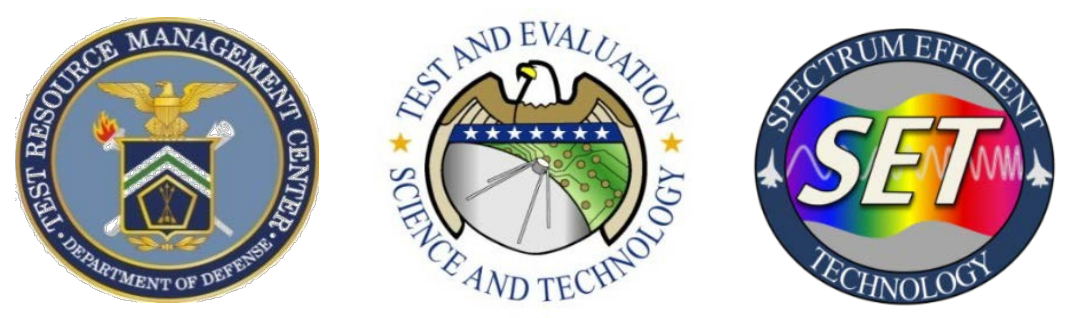

\title{
A Simulation Testbed for Adaptive Modulation and Coding in Airborne Telemetry
}

\author{
May 2014
}

Final Report

Tom Young

SET Executing Agent

412 TENG/ENI

(661) 277-1071

Email: tommy.young.1@us.af.mil

Approved for public release; distribution is unlimited.

Controlling Office: 412 TENG/ENI, Edwards AFB, CA 93524

Test Resource Management Center (TRMC) Test \& Evaluation/ Science \& Technology (T\&E/S\&T) Spectrum Efficient Technology (SET) 


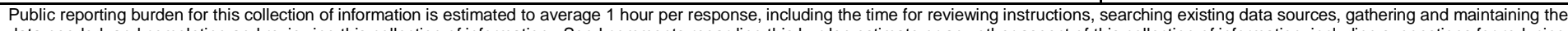

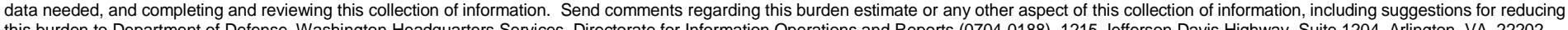

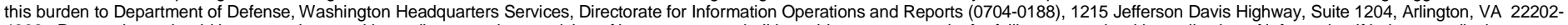

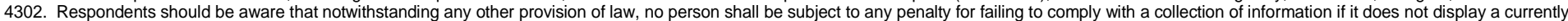
valid OMB control number. PLEASE DO NOT RETURN YOUR FORM TO THE ABOVE ADDRESS.

\begin{tabular}{l|l} 
1. REPORT DATE (DD-MM-YYYY) & 2. REPORT TYPE
\end{tabular}

29-05-2014

Technical Paper

\section{TITLE AND SUBTITLE}

A Simulation Testbed for Adaptive Modulation and Coding in Airborne Telemetry

\section{AUTHOR(S)}

Enkuang D. Wang, Brett T. Walkenhorst, Jieying Han

3. DATES COVERED (From - To)

3/13 -- 3/16

5a. CONTRACT NUMBER: W900KK- 13-C0024-P00004

5b. GRANT NUMBER: N/A

5c. PROGRAM ELEMENT NUMBER

5d. PROJECT NUMBER

5e. TASK NUMBER

5f. WORK UNIT NUMBER

8. PERFORMING ORGANIZATION REPORT NUMBER

412TW-PA-14270

10. SPONSOR/MONITOR'S ACRONYM(S)

N/A

Test Resource Management Center

Test and Evaluation/ Science and Technology

4800 Mark Center Drive, Suite 07J22

Alexandria, VA 22350

12. DISTRIBUTION I AVAILABILITY STATEMENT

Approved for public release A: distribution is unlimited.

\section{SUPPLEMENTARY NOTES}

CA: Air Force Flight Test Center Edwards AFB CA

CC: 012100

\section{ABSTRACT}

A simulation testbed has been developed that can be used as a tool for the development, implementation, and testing/verification of algorithms for airborne telemetry applications. This testbed utilizes both SOQPSK and OFDM for its modulation waveforms and LDPC for the FEC codes. It also uses several sets of published telemetry channel sounding data as its channel models. Within the context of this simulation framework, we also present an adaptive algorithm that changes a test article's modulation type and FEC code rate based on the telemetry channel quality. This paper shows the details of the simulation framework and the adaptive rules for selecting the near-optimal transmission mode. It also presents an example of an adaptive scheme that has achieved approximately 30\% to 340\% goodput performance improvement over the baseline schemes in simulation. Other potential uses of this testbed are also discussed.

\section{SUBJECT TERMS}

Spectrum, Aeronautical telemetry, algorithm, bandwidth, attached sync marker (ASM), Integrated Enhanced Networked Telemetry (iNET), Shaped Offset Quadrature Phase Shift Keying (SOQPSK), Orthogonal Frequency Division Multiplexing (OFDM), Bit Error Rate, (BER)

\begin{tabular}{|c|c|c|c|c|c|}
\hline \multicolumn{3}{|c|}{$\begin{array}{l}\text { 16. SECURITY CLASSIFICATION OF: } \\
\text { Unclassified }\end{array}$} & \multirow{2}{*}{$\begin{array}{c}\text { 17. LIMITATION } \\
\text { OF ABSTRACT } \\
\text { None }\end{array}$} & \multirow{2}{*}{$\begin{array}{l}\text { 18. NUMBER } \\
\text { OF PAGES } \\
14\end{array}$} & $\begin{array}{l}\text { 19a. NAME OF RESPONSIBLE PERSON } \\
412 \text { TENG/EN (Tech Pubs) }\end{array}$ \\
\hline $\begin{array}{l}\text { a. REPORT } \\
\text { Unclassified }\end{array}$ & $\begin{array}{l}\text { b. ABSTRACT } \\
\text { Unclassified }\end{array}$ & $\begin{array}{l}\text { c. THIS PAGE } \\
\text { Unclassified }\end{array}$ & & & $\begin{array}{l}\text { 19b. TELEPHONE NUMBER (include area } \\
\text { code) } \\
661-277-8615\end{array}$ \\
\hline
\end{tabular}




\section{DISTRIBUTION LIST}

Onsite Distribution

$\underline{\text { Number of Copies }}$

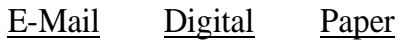

Attn. Tom Young, SET Executing Agent

0

2

2

412 TENG/ENI

61 N. Wolfe Ave. Bldg 1632

Edwards, AFB, CA 93524

Attn:Brett Walknehorst

0

Georgia Tech Applied Research Corporation

505 10TH ST

Atlanta GA 30332-0001

Edwards AFB Technical Research Libaray

0

2

2

Attn: Darrell Shiplett

307 East Popson Ave, Bldg 1400

Edwards AFB CA 93524

$\underline{\text { Offsite Distribution }}$

Defense Technical Information Center

1

0

0

DTIC/O

8725 John J Kingman Road, Suite 0944

Ft Belvoir, VA 22060-6218

U.S. ARMY PEO STRI Acquisition Center

1

0

0

Email to: kaitlin.lockett@us.army.mil

Attn: Kaitlin F. Lockett

12350 Research Parkway

Orlando, FL 32826 


\title{
A SIMULATION TESTBED FOR ADAPTIVE MODULATION AND CODING IN AIRBORNE TELEMETRY
}

\author{
Enkuang D. Wang, Brett T. Walkenhorst, and Jieying Han \\ Georgia Tech Research Institute \\ Atlanta, Georgia, USA
}

\begin{abstract}
A simulation testbed has been developed that can be used as a tool for the development, implementation, and testing/verification of algorithms for airborne telemetry applications. This testbed utilizes both SOQPSK and OFDM for its modulation waveforms and LDPC for the FEC codes. It also uses several sets of published telemetry channel sounding data as its channel models. Within the context of this simulation framework, we also present an adaptive algorithm that changes a test article's modulation type and FEC code rate based on the telemetry channel quality. This paper shows the details of the simulation framework and the adaptive rules for selecting the near-optimal transmission mode. It also presents an example of an adaptive scheme that has achieved approximately $30 \%$ to $340 \%$ goodput performance improvement over the baseline schemes in simulation. Other potential uses of this testbed are also discussed.
\end{abstract}

\section{INTRODUCTION}

Adaptive modulation and coding has been studied and implemented in several standards such as WiMAX [1], HSDPA [2], and others. These have been informed in the past by numerous academic studies over the years (see examples in [3-6]). These implementation and studies, however, are limited to single modulations compared to the multi-scheme adaptation we are investigating. Moreover, because these implementations and academic studies have not been applied to the telemetry systems, GTRI has been working to address this gap to allow telemetry systems to be more efficient in data transfer while utilizing both modulation schemes defined in the iNET standard [7]. As a first step, the authors have developed a simulation environment to develop the adaptive algorithms and verify their functionality in software. We designed the simulation software to be broadly extensible and useful for other applications and studies in the telemetry community. This paper outlines the software architecture of that environment and implementation of link adaptation within that architecture.

GTRI has developed a simulation testbed to explore the use of adaptive modulation and coding in an airborne telemetry environment. This testbed was developed based on the integrated Network Enhanced Telemetry (iNET) standard [7] and employs one of two modulation techniques: 1) shaped offset quadrature phase shift keying (SOQPSK) and 2) orthogonal frequency division multiplexing (OFDM). Both modulations have various tunable parameters, such as numbers of subcarriers, bit rates, and cyclic prefix lengths. The forward error correction (FEC) code used in this testbed is low 
density parity check (LDPC) codes with tunable code rates, and both static and dynamic telemetry channel models are included.

In an effort to maximize the aeronautical telemetry spectrum efficiency, GTRI has developed an adaptive modulation and coding scheme to be used with this simulation testbed. This adaptive scheme is different from other adaptive schemes [1-6] because it employs multi-modulation scheme adaptation, in which both SOQPSK and OFDM are used in the same adaptation rule. Based on the conditions of the channel estimate, the adaptive rule seeks to adjust the transmission parameters such that the modulation and coding scheme can tolerate the channel distortions. From the simulation results, the adaptive rule has shown significant spectral efficiency improvement in terms of total size of successful packets received (cumulative goodput) in a given simulation time.

The authors constructed the simulation testbed to be applicable to many research questions involving airborne telemetry applications other than adaptive modulation and coding. Such questions include the performance tradeoffs associated with utilizing phased array antennas at the ground station or assessing the utility of various kinds of multiple access schemes, and performance of command and control for aeronautical telemetry. These are some examples that can be facilitated by this simulation testbed by adding or modifying existing software objects and their functions while maintaining an overall structure that allows us to extract relevant results.

In Section II, we present the architecture of the simulation testbed, including the overall framework architecture, channel modeling, modulation, and demodulation. In Section III, we present a rulebased adaptive modulation and coding scheme, and its results are shown in Section IV. Other applications and uses of this simulation testbed are discussed in Section V. Conclusions are discussed in Section VI.

\section{SOFTWARE ARCHITECTURE}

\section{A. Simulation Framework Architecture}

A system model for the simulation testbed is shown in Figure 1. The testbed consists of a transmitter, a receiver, and a telemetry channel model. The transmitter has an FEC encoder, a scrambler, an attached synchronization marker (ASM) prepender, and a modulator, which includes both OFDM and SOQPSK modulations. The receiver consists of a demodulator, a decoder, and a descrambler. The channel models include static, user-defined, and dynamic channel models. The implementation and design for each of the system blocks are discussed in the following subsections.

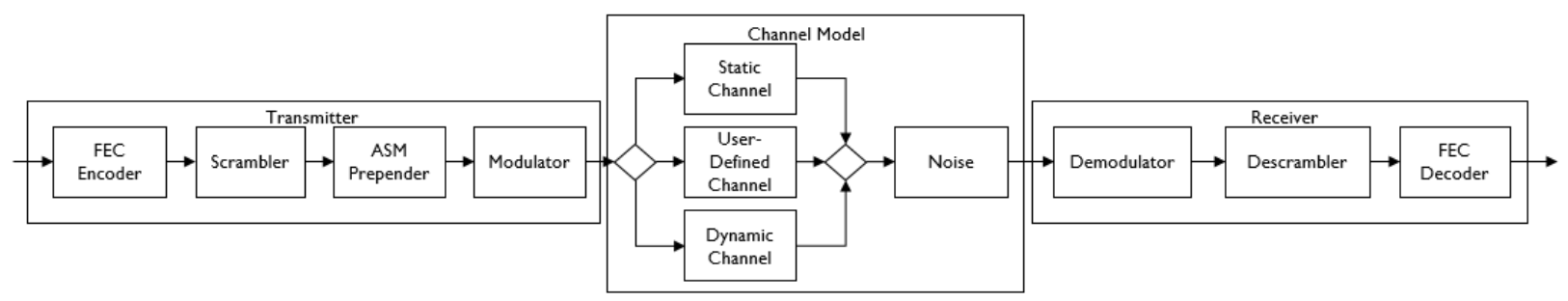

Figure 1. System model for simulation testbed.

The simulation testbed was implemented using object-oriented Matlab because we want to maximize the reuse of the objects and the flexibility of the design to other telemetry applications 
besides the adaptive schemes. The hierarchical view of classes for the system model is shown in Figure 2, and the diagram utilizes the Unified Modeling Language (UML) notation to capture the hierarchy and dependencies of each class. The dotted lines indicate dependencies, the triangle arrows indicate generalizations, arrows with an empty rhombus indicate aggregations, and arrows with a filled rhombus indicate compositions. In the diagram, darker colored boxes indicate Phase one development. Many of the boxes shown here contain multiple subclasses, but we restrict our presentation here to the top-level due to space limitation.

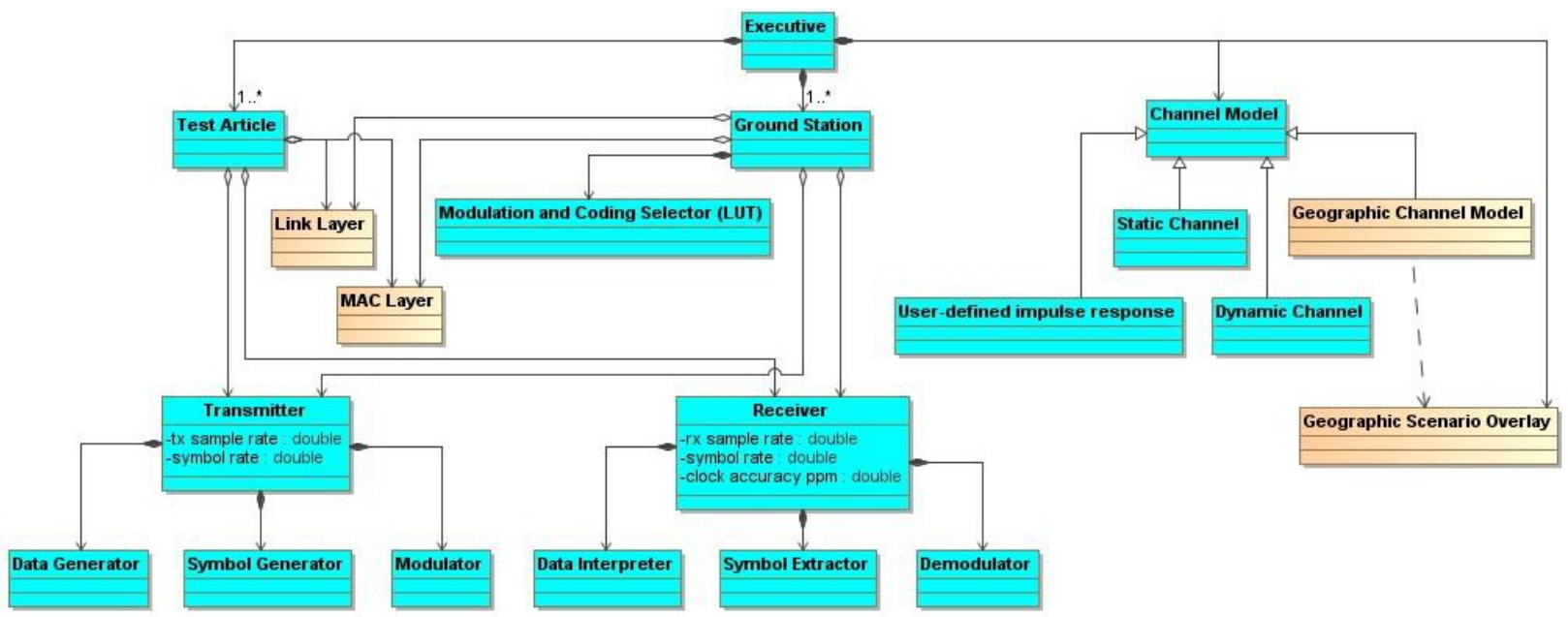

Figure 2. UML diagram showing the top-level view of the simulation testbed.

The Matlab graphic user interface (GUI) of the simulation testbed is shown in Figure 3. As seen from this figure, the user can select different modulation (SOQPSK and OFDM), coding, and channel parameters. The "Enable LDAR" check box is used for the adaptive modulation and coding scheme GTRI has developed.

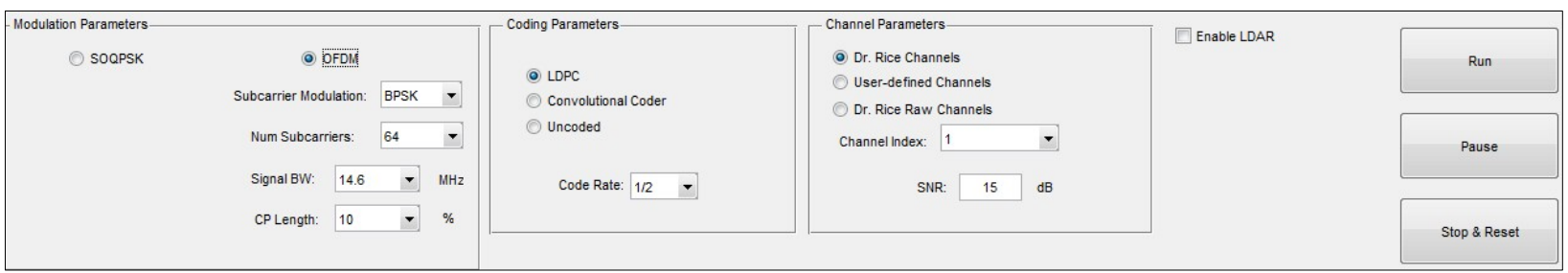

Figure 3. Simulation testbed graphical user interface (GUI) in Matlab.

\section{B. Transmitter}

\section{1) LDPC Encode}

The first task in the simulation testbed is to encode and modulate the message stream. The FEC encoder in this testbed is an LDPC encoder. LDPC codes are binary block codes with large code blocks, and the LDPC code used in the iNET standard is a 2/3 rate punctured LDPC block from [11]. Each LDPC code is specified by its parity check matrix, and the construction algorithm for

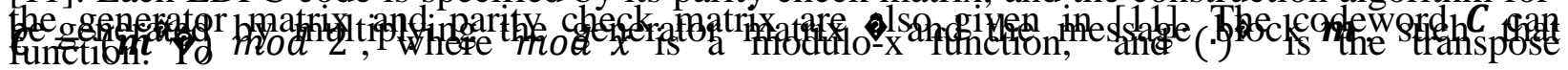


increase the flexibility of the software, we have implemented all four code rates specified in [11] for this particular LDPC code (code rates 1/2, 2/3, 3/4, and 4/5).

\section{2) Scrambler and ASM}

In addition to the LDPC code, a pseudo-randomizer is used in the simulation testbed to maximize the entropy of the data stream, enabling robust synchronization at the receiver. The codeword is scrambled by exclusive-OR (XOR) with the pseudo-random sequence from [7]. The ASM, however, is not scrambled because it is used for frame synchronization, and the ASM used in the testbed is a 64 bit pattern (034776C7272895B0hex) prepended to each codeblock frame as the synchronization header, and the burst sequence structure is shown in Figure 4 [7].

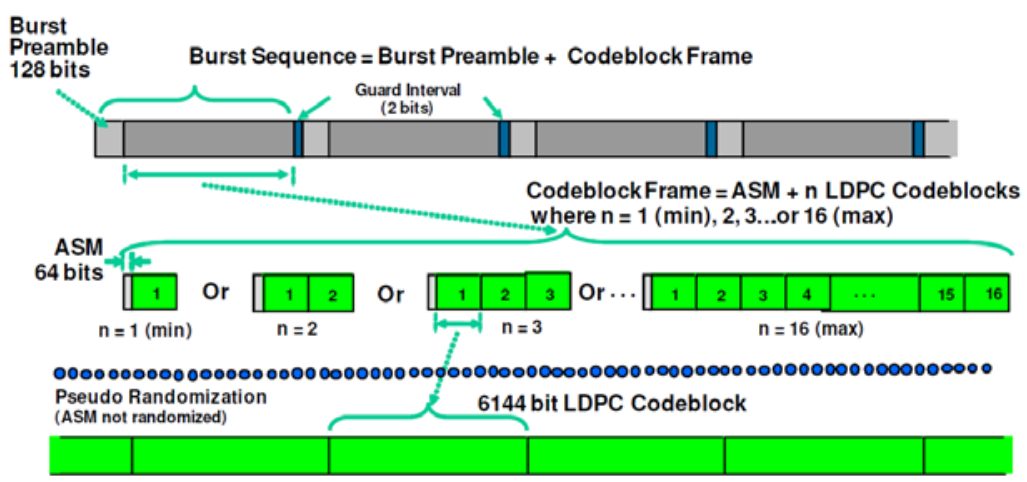

Figure 4. Burst sequence construction [7].

\section{3) OFDM Modulation}

The iNET standard specifies IEEE 802.11a [7] OFDM as the standard multi-carrier waveform with QPSK and 16 QAM as the iNET subcarrier modulation schemes. The physical layer convergence protocol (PLCP) preamble field for synchronization consists of short training symbols (STS) and long training symbols (LTS). The general modulation process for OFDM is defined in [8]. Additional tunable parameters were implemented for OFDM, including extended subcarrier modulation schemes including BPSK and 64 QAM, various number of subcarriers, and cyclic prefix lengths. The list of the tunable OFDM parameters is shown in Table 1. Due to the extended OFDM parameters, new PLCP preamble fields were implemented in our design to accommodate OFDM synchronization and channel estimation for higher orders of subcarriers. For example, since the LTS designed in the IEEE 802.11a standard was only applicable to 64 subcarriers, the LTS for higher numbers of subcarriers was implemented by repeating the same LTS in the frequency domain.

Table 1. OFDM Tunable Parameters

\begin{tabular}{|l|l|}
\hline Tunable Parameters & Values \\
\hline Subcarrier Modulation Types & BPSK, QPSK, 16QAM, 64QAM \\
\hline Cyclic Prefixes & $10 \%, 25 \%, 50 \%$ \\
\hline Numbers of Subcarriers & $64,128,256,512,1024,2048,4096$ \\
\hline
\end{tabular}

\section{4) SOQPSK Modulation}

The single carrier waveform used in this testbed is SOQPSK-TG (Telemetry Group SOQPSK) [7]. The implementation of an SOQPSK-TG modulator is shown in Figure 5. The transmitted symbol 
sequence is taken from the ternary alphabet, $\alpha \in\{-1,0,1\}$, and the frequency pulse for SOQPSK- 
TG is a product of a frequency-domain raised cosine function with a time-domain raised cosine function defined in [7]. Similar to the OFDM modulation, a 128 bit preamble is prepended to the transmitted signal, $\mathrm{s}(\mathrm{t})$, for channel equalization. In addition to the $12.5 \mathrm{Mbps}$ bit rate defined in the iNET standard, we have added four extra bit rate selections (5, 6.7, 7.5, and $10 \mathrm{Mbps}$ ) for SOQPSK modulation to increase the flexibility of the design.

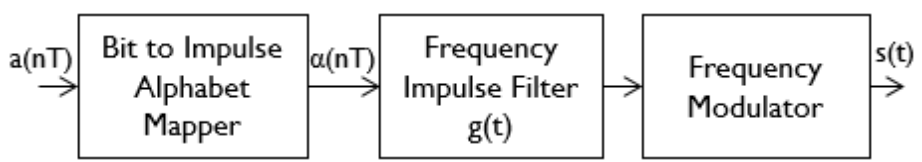

Figure 5. SOQPSK-TG modulator block diagram [5].

\section{Channel Models}

After the input message is encoded, scrambled, and modulated, the baseband waveform is filtered with a channel model to simulate the effects of a wireless air-ground telemetry channel on the transmitted signal. As shown in Figure 1, three types of channel models are used in the testbed: static, user-defined, and dynamic channel models. The user can also pick a desired signal-to-noise ratio (SNR) in $\mathrm{dB}$.

\section{1) Static Channel Models}

The static channel model consists of 11 different static telemetry channel models identified by Dr. Michael Rice's telemetry lab as representative of the telemetry environment over Edwards Air Force Base [15]. These channel models were used as the representative channels for our adaptive modulation and coding scheme simulation.

\section{2) User Defined Channel Models}

The user-defined channel model option in the simulator allows the user to input their desired models. The input format for this channel model is the channel impulse response. For example, to get the impulse response and frequency response of a three-ray model in [9], the user would input the impulse response as $\left[1,0.88 \mathrm{e}^{\mathrm{j} 1.3}, 0,0,0,0,0.23 \mathrm{e}^{-\mathrm{j} 2.0}\right]$ since the default sampling rate of the user-defined channel is $20 \mathrm{MHz}$. Similarly, to get an additive white Gaussian noise (AWGN) channel, the input for the impulse response is simply 1.

\section{3) Dynamic Channel Models}

The dynamic channel models that are used in this testbed are taken from [14]. This set of channel models consists of data collected over different flight paths, including flights approaching and moving away from a ground station, various TARMAC paths, and takeoff and landing models. The sampling rate of the collected data set is $200 \mathrm{MHz}$. All of the dynamic channel models were trimmed using a non-linear filter (where only the samples that contributed to $90 \%$ of the total energy were kept and the rest were zeroed), normalized to unit energy, and shifted to $0 \mathrm{~Hz}$ center to eliminate carrier frequency offset (CFO) and compensate for the centroid of the Doppler shift.

\section{Receiver}

After the transmitted signal is filtered with a channel model, the filtered signal arrives at the receiver. The receiver is responsible for demodulating, descrambling, and decoding the received 
signal. Channel and link metrics including error vector magnitude (EVM) [8], dispersion [13], bit error rate (BER) are also computed at the receiver.

\section{1) OFDM Demodulation}

The process of the OFDM demodulation is defined in [8]. The channel estimate for OFDM is computed using the two received LTS blocks preceding the codeword and the known LTS via a least square (LS) method [16]. The LS method is defined as

$$
\hat{Q}=\vartheta^{-1}
$$

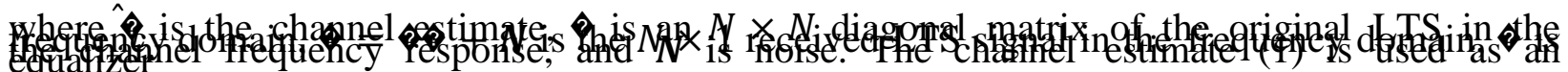
to correct all of the OFDM signals with a simple multiplication. As previously mentioned, the STS of the PLCP is yet to be used in the testbed because CFO or phase offset has not been introduced to the transmitted signals, though they may be added in future work.

\section{2) SOQPSK Demodulation}

In our development effort thus far, the SOQPSK demodulator is very simple because only a timedomain equalizer is used to estimate the channel response. No timing, frequency, or phase offset detection is included because none of these offsets have been introduced in the testbed. The equalizer for SOQPSK demodulator is a minimum mean-squared error (MMSE) filter [10]

$$
\checkmark A^{-1}
$$

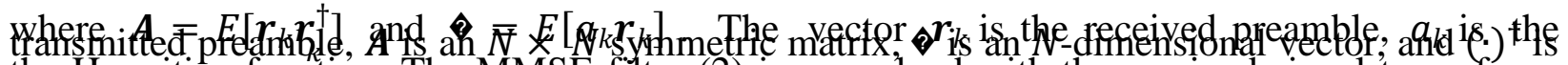
the Hermitian function. The MMSE filter (2) is convolved with the received signal to perform equalization.

\section{3) Descrambler}

As shown in Figure 4, an ASM is attached before each codeblock to aid the decoder in detecting and resolving phase ambiguities. The descrambling process happens after demodulation and before the decoder, and the process is the same as the scrambling process in [7].

\section{4) LDPC Decode}

The LDPC decoder is specified by its parity check matrix H. The inputs of the decoder are log likelihood ratios (LLR) of the received signals from the demodulators. The decoder algorithm used in the testbed is specified in [12], in which $\mathrm{H}$ is used to check whether or not the parity check equation is satisfied. The parity check equation is given as

$$
\diamond=0
$$

where is the parity check matrix, is the codeword, and $(\cdot)$ is the transpose function. If (3) is not satisfied, the decoder continues to decode until either the equation is satisfied or the maximum iteration is finished. 


\section{LINK DEPENDENT ADAPTIVE RADIO}

Using this simulation testbed, GTRI has developed an adaptive scheme that can adapt across two modulation schemes: SOQPSK and OFDM. We call the adaptive algorithm link dependent adaptive radio (LDAR) because the adaptability of the radio depends on the telemetry link quality. This algorithm selects a near-optimal transmission mode based on the highest throughput available among the various combinations of modulation and coding parameters while ensuring a minimum level of reliability. The selection of transmission parameters is based on the channel characteristics with metrics including RMS delay spread of the channel and EVM for OFDM (or dispersion [13] for the SOQPSK) received signal. The adapter in the GS conveys the chosen transmission mode to the TA. The TA encodes and modulates the message using the desired transmission parameters.

The adaptation rules used in this scheme were developed empirically from several simulation runs with the 11 static channel models. For each static channel, a lookup table (LUT) was created during the simulations, and it includes a collection of the values of EVM, dispersion, BER, throughput, and goodput for various SNRs. In addition, since each of the static channel models has a unique RMS delay spread, LDAR chooses a channel with the closest RMS delay spread from the static models as the representative for the current channel model. Then, the current EVM/dispersion value is compared to the table $\mathrm{EVM}$ /dispersion values with the same transmission parameters. If the current EVM/dispersion value is less than the LUT value, and the LUT BER for the same parameters is less than the BER threshold, the transmission parameters with the highest throughput is selected as the new transmission scheme. The BER threshold for the LUT is chosen as $1 \times 10^{-5}$. In other words, we don't allow a rule to select a transmission scheme that would result in a BER higher than $1 \times 10^{-5}$. The algorithm for LDAR is summarized below.

LDAR Algorithm for Selecting New Transmission Mode

1: Compute the RMS delay spread of the current channel

2: Select a static channel that has the closest RMS delay spread as the representative channel

3: Look up the EVM/Dispersion value with the same transmission mode from the LUT of the representative channel

4: Select a mode with the highest throughput that has a BER lower than the threshold 5: Repeat Steps 1 to 4 for each delivered packet

\section{SIMULATION RESULTS}

In this section, we show simulation results for the LDAR algorithm alongside a baseline modulation. The baseline scheme we use is an OFDM waveform with 2/3 code rate and 16 QAM subcarrier modulation. The scheme was chosen because it has the highest throughput among the three potential baseline schemes (2/3 SOQPSK, 2/3 QPSK, and 2/3 16 QAM) specified in [7]. The dynamic channel models are used for the simulations. From the simulation result, LDAR has significantly better goodput performance compared to the baseline scheme. A comparison between the cumulative goodput of the two schemes is summarized in Table 2.

As shown from Table 2, the performance increases range from 30 to $120 \%$. Since the data rate for 2/3 16 QAM (28.8 Mbps) is twice as much as the data rate of 2/3 QPSK (14.4 Mbps) and 2/3 SOQPSK (12.5 Mbps), the cumulative goodput performance increase of the LDAR scheme to each 
of these schemes is approximately $130 \%$ to $340 \%$. Moreover, for some frequency selective fading channels (channels 3, 6, and 8), none of the packets of the baseline scheme were delivered. The LDAR scheme, however, was able to successfully deliver packets by adaptively selecting transmission schemes to avoid loss of data. The cumulative goodput plots of the selected baseline scheme and the LDAR scheme of channels 2, 7, and 11 are shown in Figure 7.

Table 2. Cumulative Goodput Performance Comparison

\begin{tabular}{|l|l|l|l|}
\hline $\begin{array}{l}\text { Dynamic } \\
\text { Channel }\end{array}$ & $\begin{array}{l}\text { Baseline } \\
\text { Goodput (Mb) }\end{array}$ & $\begin{array}{l}\text { LDAR } \\
\text { Goodput (Mb) }\end{array}$ & $\begin{array}{l}\text { Performance } \\
\text { Increase (\%) }\end{array}$ \\
\hline 1 & 4.0 & 5.2 & 30 \\
\hline 2 & 2.9 & 5.3 & 83 \\
\hline 3 & 0 & 4.2 & N/A \\
\hline 4 & 2.0 & 4.4 & 120 \\
\hline 5 & 4.0 & 8.2 & 105 \\
\hline 6 & 0 & 8.1 & N/A \\
\hline 7 & 3.3 & 4.4 & 33 \\
\hline 8 & 0 & 3.7 & N/A \\
\hline 9 & 3.3 & 5.2 & 58 \\
\hline 10 & 4.0 & 8.8 & 120 \\
\hline 11 & 4.0 & 8.4 & 110 \\
\hline
\end{tabular}

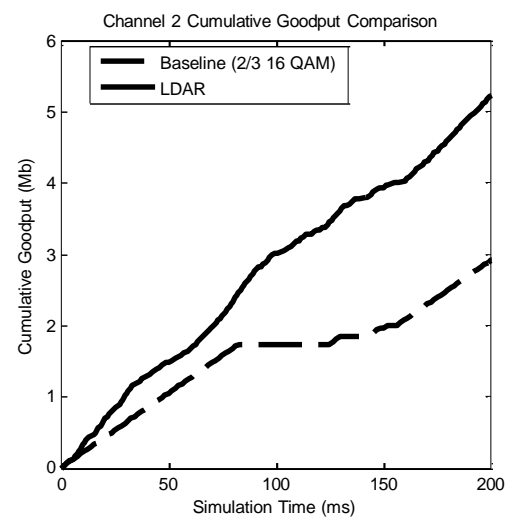

(a)

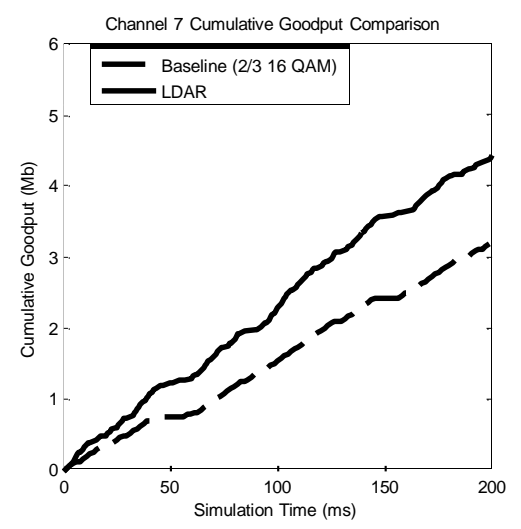

(b)

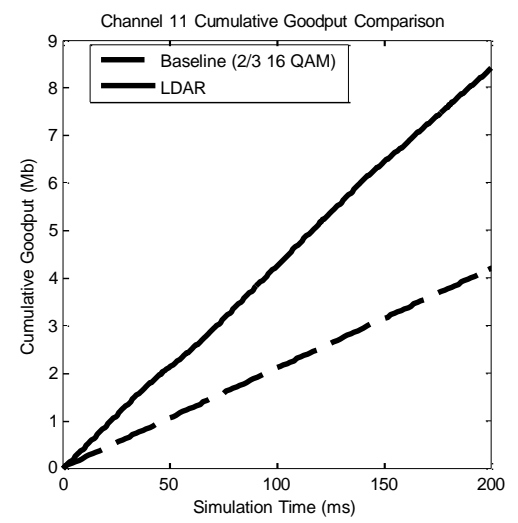

(c)

Figure 7. Cumulative goodput comparisons of the baseline and LDAR schemes on channel 2 (a), channel 7 (b), and channel 11 (c).

Channel 2 is one of the frequency selective fading TARMAC channel models. As illustrated in Figure 7 (a), all of the packets of the baseline scheme were dropped for approximately $40 \mathrm{~ms}$ (between 80 and $120 \mathrm{~ms}$ ) whereas the LDAR scheme was able to adapt its transmission parameters such that it would continually deliver packets during this period. As shown in Table 2, the goodput performance increase for channel 7 is the lowest. This is because channel 7 is very inconsistent, in which the channel impulse response is constantly changing and largely uncorrelated from one frame to the next. Because LDAR selects a scheme based on the previous frame, if the frames are changing rapidly enough, the selected transmission scheme may not work in the new frame. The same scheme, however, may work if consecutive frames are correlated. As shown in Figure 7 (b), the LDAR scheme is not particularly robust in this channel, but it can still outperform the baseline 
scheme. Finally, channel 11 is very consistent and smooth. This can be illustrated in Figure 7 (c), in which the cumulative goodput of the LDAR and baseline schemes increase consistently throughout the entire simulation period. This shows that LDAR would constantly pick the transmission scheme with the highest throughput (4/5 code rate and 64 QAM) if the channel frames are smooth.

\section{OTHER APPLICATIONS}

From the previous sections, we have shown that the simulation testbed can be used to simulate an adaptive modulation and coding scheme for an airborne telemetry system. Other potential applications include the simulation and implementation of multiple access, phased array antennas, and command and control schemes. The simulation testbed can be reused for all of the applications since the transmitter and receiver both already possess the capability to generate the transmission signals specified in the iNET standard [7]. For the multiple access schemes, additional classes in the medium access control (MAC) layer are needed to assign specific time or frequency to the TAs, but the receiver and transmitter of the testbed can be reused for both the TA and the GS.

The same implementation structure can also be applied to phased array antennas at the GS and command and control for aeronautical telemetry. For example, for phased array antennas, the geographic channel model class, shown in Figure 2, can be added to track the locations of the TAs such that the GS will be able to adjust its antenna beam angles based on the TA position. Another addition to the testbed may be a class that computes the link budgets and SNRs for the phased array antennas since the received signal power is different for each TA. For command and control, one example the framework will need is an uplink using the same transceiver structure. Ultimately, the simulation testbed can serve as a basis for further experimentation, simulation, and research on a variety of topics associated with aeronautical telemetry.

\section{CONCLUSION AND FUTURE WORK}

A simulation testbed for aeronautical telemetry has been developed. In addition to the modulation schemes and LDPC code specified in the iNET standard, more modulation parameters and coding rates are also implemented in this testbed. The testbed is used as a tool to simulate and verify an adaptive modulation and coding scheme. The algorithm to select near-optimal transmission parameters and the results of this adaptive scheme are also discussed.

The next development phase of this testbed is to implement the classes shown in white colored blocks from Figure 2, such as the link and MAC layers. Some other classes to be implemented include CFO, Doppler shift, an iNET uplink, etc. These additional features would further improve the accuracy of the simulation testbed by simulating the telemetry environment in which the TAs are traveling at high velocities. The LDAR scheme can also be improved by adding more static channels for data collection. Since LDAR is currently using data collected from 11 static channels, the selection of different RMS delay spread is currently limited. A potential improvement to LDAR is to utilize a single channel metric to drive adaptation across both EVM and dispersion. In [17], a mathematical relationship between EVM and dispersion has been derived, allowing us to map a 
single channel metric to drive adaptation across both SOQPSK and OFDM. This may be a further enhancement of LDAR.

\section{REFERENCES}

[1] LAN/MAN Standards Committee, "IEEE Standards for Local and Metropolitan Area Network: Part 16: Air Interface for Fixed Broadband Wireless Access Systems,” IEEE Computer Society and IEEE Microwave Theory and Techniques Society, May 2004.

[2] 3GPP TR 25.858 V1.0.04, Jan 2002.

[3] Sampei, S. and Harada, H., "System design issues and performance evaluations for adaptive modulation in new wireless access systems," Proceedings of the IEEE, Vol. 95, No. 12, pp. 2456-2471, 2007.

[4] Goldsmith, A. and Chua, S. G., “Adaptive coded modulation for fading channels,” IEEE Transactions on Communications, Vol. 46, No. 5, pp. 595-602, 1998.

[5] Svensson, A., "An Introduction to Adaptive QAM Modulation Schemes for Known and Predicted Channels,” Preceedings of the IEEE, vol. 95, no. 12, pp. 2322-2336, 2007.

[6] Chung, S. T. and Goldsmith, A., "Degrees of Freedom in Adaptive Modulation: A Unified View,” IEEE Trans. On Communications, vol. 49, no. 9, pp.1561-1571, Sept 2001.

[7] integrated Network Enhanced Telemetry (iNET) Radio Access Network Standards Working Group, "Radio access network (RAN) standard, version 0.7.9," Tech. Rep., [Online] (Available: https://www.tena-sda.org/display/INET/iNET+Platform+Interface+Standards.

[8] IEEE Std 802.11a, "Supplement to Part 11: Wireless Lan Medium Access Control (MAC) and Phaysical Layer (PHY) Specifications: High-Speed Physical Layer in the $5 \mathrm{GHz}$ Band," 1999.

[9] Rice, M., Davis, A., and Bettweiser, C., “A Wideband Model for Aeronautical Telemetry”, IEEE Transactions on Aerospace and Electronic Systems, vol. 40, pp. 57-69, January 2004.

[10] Godard, D., "Channel Equalization Using a Kalman Filter for Fast Data Transmission," IBM Journal of Research and Development, vol.18, no.3, pp.267,273, May 1974

[11] Consultative Committee for Space Data Systems, "Low Density Parity Check Codes for Use in Near-Earth And Deep Space Applications," September 2007, [Online] (Available: http://public.ccsds.org/publications/archive/131x1o2e2.

[12] Moon, T., Error Correction Coding: Mathematical Methods and Algorithms. New York: Wiley-Interscience, 2005.

[13] Godard, D., "Self-recovering equalization and carrier tracking in two-dimensional data communication systems,” IEEE Trans.Commun., vol. 28, no. 11, pp. 1867-1875, Nov. 1980.

[14] Rice, M. "Final Report: Multipath Modeling and Mitigation using Multiple Antennas (M4A),” Brigham Young University, Provo, UT, 2013.

[15] Rice, M. "Phase 1 Final Report: Preamble Assisted Equalization for Aeronautical Telemetry (PAQ),” Brigham Young University, Provo, UT, 2014,

[16] van de Beek, J.-J., Edfors, O., Sandell, M., Wilson, S.K., and Ola Borjesson, P., "On channel estimation in OFDM systems," Vehicular Technology Conference, 1995 IEEE 45th , vol.2, pp.815-819, July 1995

[17] Han, J., Walkenhorst, B., and Wang, E., "Adaptive Modulation and Coding Schemes for OFDM and SOQPSK Using Error Vector Magnitude (EVM) and Godard Dispersion,” pending publication in Proceedings of the International Telemetering Conference, San Diego, CA, Oct. 2014. 